\title{
Reverse shoulder arthroplasty patient personalized rehabilitation protocol. Preliminary results according to prognostic groups
}

\author{
Alfonso Maria Romano ${ }^{1}$ \\ Francesco Oliva $^{2}$ \\ Guglielmo Nastrucci ${ }^{1}$ \\ Pasquale Casillo ${ }^{3}$ \\ Angelo Di Giunta ${ }^{4}$ \\ Massimiliano Susanna ${ }^{5}$ \\ Francesco Ascione $^{3}$ \\ 1 Orthopedic Division, Campolongo Hospital, Salerno, \\ Italy \\ 2 Department of Orthopaedics and Traumatology, \\ University of Rome "Tor Vergata", Rome, Italy \\ 3 I Orthopedic and Traumatology Unit, Università \\ della Campania "L. Vanvitelli", Napoli, Italy \\ 4 Orthopaedic Division of Policlinico "G.B. Morgagni", \\ Catania, Italy \\ 5 Orthopedic and Traumatology Unit, San Donà \\ di Piave Hospital, Venezia, Italy
}

Corresponding author:

Francesco Ascione

I Orthopedic and Traumatology Unit,

Università della Campania "L. Vanvitelli"

Vico Luigi De Crecchio

80138 Napoli, Italy

E-mail: dr.francesco.ascione@gmail.com

\section{Summary}

Background: Rehabilitation after Reverse Shoulder Arthroplasty (RSA) is still object of discussion with no based evidence guidelines. The aim of this study was to test a personalized rehabilitation protocol for three different patients groups according to clinical and intraoperative parameters that can affect final outcomes after RSA.

Patients and methods: We retrospectively evaluated a consecutive series of 112 patients who underwent to RSA between 2013 and 2015 . The average follow-up was 29.2 months. A preoperative and postoperative clinical and radiographic assessment was performed. According to clinical, radiographic and intraoperative parameters, we selected three groups dedicating each one a specific level of post surgical care.

Results: A statistically significant improvement was achieved from preoperative evaluation, regarding all analysed parameters. Notable improvements were reported in high care group: elevation improved of $63.9^{\circ}$ despite of Group A $55.5^{\circ}$ and Group $B 54.5^{\circ}$ and it resulted statistically significant $(p<$ 0.05). Costant Score increased of $35.9 \%$, despite of Group A and Group B respectively $40.6 \%$ and $34.8 \%$ ( $p>0.05)$.

Conclusion: Our study shows that a personalized rehabilitation protocol can be effective improving clinical outcomes and decreasing complications rate, particularly in difficult management patients. Level of Evidence: Therapeutic Level IV.

KEY WORDS: complications, onlay design, radiological results, rehabilitation protocol, reverse shoulder arthroplasty, scapular notching.

\section{Introduction}

Reverse shoulder arthroplasty (RSA) is an increasing procedure in orthopedic surgery. Recently, indications became wider allowing the management of different shoulder diseases. RSA, firstly was indicated for pseudoparalitic shoulders with massive rotator cuff tears (MRCT); has been published that RSA may have good results even in acute fractures in aged patients, rotator cuff tears arthropathy (CTA), primary osteoarthritis (OA), gleno-humeral rheumatoid arthritis (RA), fractures sequelae and, in glenoid excessive retroversion and humeral head static subluxation in good cuff status shoulders ${ }^{1-9}$, resulting in significant and predictable pain relief and restoration of active elevation by the medialization of the center of rotation and lowering of the humerus, with durable efficiency ${ }^{1}$. Due to modified biomechanics and constraints of gleno-humeral joint, complications after RSA seem to be about 4-fold more than anatomic total shoulder arthroplasty and hemiarthroplasty at mid-term follow up ${ }^{10-12}$. Validated rehabilitation programs after RSA are lacking of evidence, only expert opinions are present in literature ${ }^{13-15}$.

Different diagnoses, preoperative patient activity, muscles status, bone assessment of glenoid, intraoperative variables and complications, such as implant features or iatrogenic peri-prostheses fractures, subscapularis tendon repair technique are the much important variables which make arduous to establish universal parameters for rehabilitation protocol. In our opinion, to dedicate a patient-specific rehabilitation protocol can considerably improve clinical results and decrease complications of RSA. 
Therefore, the aim of this study was to verify a personalized rehabilitation protocol, setting patients in three different groups according to preoperative and intraoperative parameters, which can affect (positively or negatively) final functional outcomes and incidence of complications after RSA on the basis of current literature.

\section{Patients and methods}

Between 2013 and 2015, a consecutive series of 112 patients were submitted to a RSA operation by one of us (AMR). There were 83 females $(74 \%)$ and 29 males $(26 \%)$. The average age at surgery was 72.2 years (range, 60-87). The average duration of followup after surgery was 29.2 months (range, 12-36 months). Every patient had a preoperative and postoperative clinical and radiological evaluation, active anterior elevation (AAE), active external rotation arm at side (ER1), active internal rotation hand level in points (IR1), Constant Score (CS) were recorded at 2 weeks, 1, 3 and 6 months, at 1, 2, 3 years consultations.

All patients were investigated with a true AP and axillary view X-ray images and CT scan preoperatively. $X$-ray exams were carried out at the immediate postoperative, at 1 months, 6 months and then yearly. Preoperative CT images were settled according to ordinary classification systems: glenoid Walch ${ }^{16}$ and Favard ${ }^{17}$ staging, Goutallier ${ }^{18}$ muscle fatty infiltration grades, Levigne ${ }^{19}$ shoulder Rheumatoid Arthritis. All radiographs were also analyzed for tuberosities resorptions, glenohumeral ossifications, scapular spurs and scapular notching according to Sirveaux-Nerot classification ${ }^{8}$. Mechanical problems and complications as: implant loosening, subsidence, disassembly, fracture, instability, migration, tuberosities status, radiolucent lines (RLL) around the stem and the glenoid components in postoperative and last follow-up radiographs were recorded.

\section{Inclusion criteria}

Rotator cuff arthropathy (CTA), primary osteoarthritis (OA), massive rotator cuff tear (MRCT), fracture sequelae, total or hemi arthroplasty revision surgery.

\section{Exclusion criteria}

Acute fractures and neurological associated diseases.

\section{Patients Groups features}

According to clinical diagnosis, radiographic preoperative data and intraoperative parameters that can lead to positive or negative results or facilitate the incidence of complications, we selected three groups of patients, dedicating each one a specific level of care, as follows 2 , 6, 9, 12, 15- 25 .
Group A (low care, $n=52$ ): diagnosis of Primary OA, CTA, massive rotator cuff tear, absence of hyperkyphosis or scapular dyskinesia, Glenoid assessment according to Walch ${ }^{16} \mathrm{~A} 1$ or $\mathrm{A} 2$ and according to Favard $^{17}$ E0, E1 and E2, Infraspinatus and Teres minor fatty infiltration according to Goutallier ${ }^{18,22}$ less than 3, no spine nonunion, not repaired SSC, no intraoperative complications or tendon transfers.

Group B (medium care, $n=39$ ): patients not included in Group A and C.

Group C (high care, $\mathrm{n}=21$ ): diagnosis of Arthroplasty Revision surgery, Fracture sequelae, Rheumatoid arthritis (grade 3 according to Levigne ${ }^{19}$ ) or/and intraoperative complications or/and tendon transfers associated procedures.

Rehabilitation protocol adopted for each group is summarized in Table I.

\section{Surgical procedure}

All patients underwent surgery in beach chair position using a delto-pectoral approach. The prosthesis implanted was Equinoxe Reverse prosthesis (Exactech Inc, Gainesville, FL, USA).

A tenotomy of the subscapularis was made on the lesser tuberosity, superiorly, from the rotator interval to the inferior one-third of the subscapularis. The subscapularis tendon and anterior capsule were released as a single unit. The labrum was removed completely and glenoid was circumferentially anteriorly exposed in all cases. The meta-glenoid baseplate was placed as far inferior as necessary, with the inferior offset of $2 \mathrm{~mm}$.

The humeral shaft was prepared by first reaming distally using sequential reamers. The proximal humerus was broached placing the component in $20^{\circ}$ of retroversion. That component was then placed into place once the tension on the system had been tested and found to be stable. Subscapularis tendon was repaired in slight abduction and external rotation, when possible. All incisions were closed over a suction drain.

\section{Statistical analysis}

Statistical analysis was performed using the Social Science Statistics collaborative web site (http://www.socscistatistics.com). Statistical analysis included demographic analysis and also comparison of parametric measures using the Student t test. A $p$ value $<0.05$ was considered significant.

\section{Results}

The Group A counts 53 patients (47.3\%), the Group B 39 patients $(34.8 \%)$ and the Group C 20 patients (17.9\%). Clinical results are showed in Table II: they show a statistically significant improvement from preoperative situation, regarding all analyzed parameters 
Reverse shoulder arthroplasty patient personalized rehabilitation protocol. Preliminary results according to prognostic groups

Table I. Rehabilitation protocols according to groups.

\begin{tabular}{|c|c|c|}
\hline Group A & Group B & Group C \\
\hline $\begin{array}{l}\text { A slight abduction sling for } 2 \text { weeks; the } \\
\text { day after allowed to recover the passive } \\
\text { ROM (active exercises of the elbow, wrist } \\
\text { and hand). }\end{array}$ & $\begin{array}{l}\text { Similarly to Group A (dedicating a } \\
\text { physiotherapist } 3 \text { times weekly for } 4 \\
\text { months and slight abduction sling } \\
\text { for } 3 \text { weeks). }\end{array}$ & $\begin{array}{l}\text { In addition to Group } A \text { and } B \text { protocol, } \\
\text { a fully trained physio therapist team } \\
\text { follows the patient every day for } 2 \\
\text { months in case of fracture sequelae } \\
\text { and for } 1 \text { month in the other cases. }\end{array}$ \\
\hline $\begin{array}{l}\text { From 2nd week active movements } \\
\text { assisted can be started (flexion up to } 60^{\circ} \text {, } \\
\text { abduction up to } 45 / 60^{\circ} \text {, ER in the scapular } \\
\text { plane up to } 20^{\circ} \text { ). }\end{array}$ & $\begin{array}{l}\text { Immediate postoperative, focus on } \\
\text { scapula-thoracic joint trying to } \\
\text { restore full range of motion, freeing } \\
\text { adherences and at } 3^{\text {rd }} \text { week stren- } \\
\text { gthening stabilizer muscles }\end{array}$ & $\begin{array}{l}\text { Then } 3 \text { times a week until a total of } 5- \\
6 \text { months assisted therapy, slight } \\
\text { abduction sling for } 3-4 \text { weeks. }\end{array}$ \\
\hline $\begin{array}{l}\text { From the } 3^{\text {rd }} \text { week, consolidate all the } \\
\text { previous advances and gradually adding } \\
\text { isometric exercises for scapula stabilizing } \\
\text { muscles and deltoid, proprioception exer- } \\
\text { cises (flexion up to } 120^{\circ} \text {, abduction up to } \\
90^{\circ} \text {, ER } 20^{\circ} \text {, beginning with IR with } 60^{\circ} \\
\text { abduction). } \\
\text { From } 6^{\text {th }} \text { to } 8^{\text {th }} \text { week, passive and active- } \\
\text { assisted mobilization (flexion } 130^{\circ}-180^{\circ} \text {, } \\
\text { gradual increase of ER } 1 \text { and IR } 1 \text { ). }\end{array}$ & $\begin{array}{l}\text { From } 2^{\text {nd }} \text { week (extending all the } \\
\text { phases to } 1 \text { month more compared } \\
\text { first group), muscle electrostimu- } \\
\text { lation therapy for the remaining } \\
\text { cuff, posterior deltoid fibers and the } \\
\text { external rotation muscles; isometric } \\
\text { exercises for external rotation. }\end{array}$ & $\begin{array}{l}\text { We often help this group of patients } \\
\text { with frequent physician consultations } \\
\text { in which we establish exact objectives } \\
\text { at a certain time. }\end{array}$ \\
\hline $\begin{array}{l}\text { At } 8^{\text {th }} \text { week exercises with elastic bands to } \\
\text { strengthen muscles, until the } 12^{\text {th }} \text { week. } \\
\text { (The therapist seeks every full recovery } \\
\text { aspect: the active and passive ROM, } \\
\text { stability, joint dynamic, fluidity of move- } \\
\text { ment and neuromuscular coordina-tion, } \\
\text { aiming to reach daily activities and return } \\
\text { to previous job). }\end{array}$ & $\begin{array}{l}\text { In case of SSC tendon-to-tendon } \\
\text { repair, passive external rotation } \\
\text { forbidden for the } 1 \text { st month; active } \\
\text { internal rotation is begun never } \\
\text { before the } 2^{\text {nd }} \text { month. } \\
\text { Following Group A indications. }\end{array}$ & \\
\hline
\end{tabular}

(excepted the Group B and C internal rotation improvement). Obviously, considered the worst preoperative shoulder status, high improvements were reported in Group C (Fig. 1): at final follow-up examination, in this Group AAE improved of $63.9^{\circ}$ despite of Group A $55.5^{\circ}$ and Group B $54.5^{\circ}$ and it resulted statistically significant $(p<0.05)$. Total CS increased of $35.9 \%$, despite of Group A and Group B respectively $40.6 \%$ and $34.8 \%(p>0.05)$.

\section{Radiological results and complications}

Scapular notching was detected at last follow-up in 31 radiographs $(27.7 \%)$, but the grade was never beyond the 2 according to Sirveaux-Nerot classification $^{8,24}$. In $24.1 \%$ of cases it was staged as 1 and $3.6 \%$ as 2. Notching was showed in 12 of Group A (23.1\%), 10 of Group B (25.6\%) and 9 of Group C (42.9\%) cases (Fig. 2).

Humeral stress shielding was present at last radiological assessment in $5.4 \%$ of cases, appearing in proximal bone narrowing or osteopenia ( $3,1,2$ cases in Group A, B, C respectively). No radiological radiolucent lines were found around glenoid and humeral stem.

An intraoperative periprosthetic fracture of the proximal metaphysis was developed in 1 case $(0.8 \%)$ in the Group $\mathrm{C}$ and simple cerclage wiring around the fracture was performed.
Complications reported were one dislocation (0.8\%) in Group C patients and two early infections (1.6\%) in Group B, treated successfully by implant insert changes and debridement.

The dislocation was treated by reduction and ticker polyethylene insert substitution.

No cases of revision were found.

\section{Discussion}

Our patient personalized rehabilitation protocol leads to good results and an inferior percentage of compli-

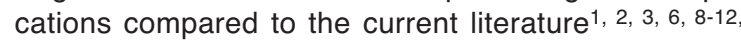
$23,24,26$, even if we have only preliminary results.

After the RSA surgery, patients immediately start the rehabilitation phase with protocols aimed to the achievement of joint function, weighted according to risk groups (A-B-C Group).

All patients wear after surgery a sling in abduction of $15-30^{\circ}$, at least four weeks. After 7 days, patients who belong to Group A and B wear the sling only in the night and keep the limb free for most of the day. Early mobilization and sling discharge is considered primary in our protocol to reach optimal clinical results (Fig. 1).

The initial limit of passive external rotation allowed is based on the quality of the subscapularis repair and 
Table II. Clinical results of each group.

\begin{tabular}{|c|c|c|c|c|}
\hline Group A (n 52) & Preoperative & Postoperative & $\Delta$ & $p$ \\
\hline AAE & $89.2^{\circ}$ & $144.7^{\circ}$ & 55.5 & $<0.001$ \\
\hline ER1 & $8.6^{\circ}$ & $26.5^{\circ}$ & 17.9 & $<0.001$ \\
\hline IR1 (0-10) & 3.2 & 7.2 & 4 & $<0.05$ \\
\hline CS Pain /15 & 4.3 & 13.8 & 9.5 & $<0.01$ \\
\hline CS $\%$ & 29.5 & 70.1 & 40.6 & $<0.001$ \\
\hline Group B (n 39) & Preoperative & Postoperative & $\Delta$ & $p$ \\
\hline AAE & $78.3^{\circ}$ & $132.8^{\circ}$ & 54.5 & $<0.001$ \\
\hline ER1 & $2.3^{\circ}$ & $12.6^{\circ}$ & 10.3 & $<0.01$ \\
\hline IR1 (0-10) & 3.8 & 5 & 1.2 & $>0.05$ \\
\hline CS Pain /15 & 4.6 & 12.9 & 8.3 & $<0.01$ \\
\hline CS $\%$ & 28.7 & 63.5 & 34.8 & $<0.001$ \\
\hline Group C (n 21) & Preoperative & Postoperative & $\Delta$ & $p$ \\
\hline AAE & $58.9^{\circ}$ & $122.8^{\circ}$ & 63.9 & $<0.001$ \\
\hline ER1 & $-4.5^{\circ}$ & $7.9^{\circ}$ & 12.4 & $<0.01$ \\
\hline IR1 (0-10) & 2.1 & 4.3 & 2.2 & $>0.05$ \\
\hline CS Pain /15 & 3.7 & 11.6 & 7.9 & $<0.05$ \\
\hline CS \% & 23.5 & 59.4 & 35.9 & $<0.001$ \\
\hline
\end{tabular}

Abbreviation: AAE, Active Anterior Elevation; ER1, External Rotation with elbow at side; IR1, Internal Rotation hand level in points; CS, Constant Score.

the amount of passive external rotation obtained intraoperatively. This limitation is adhered to for a period of 4 to 6 weeks.

The recovery of a patient undergone to a reverse prosthesis is closely linked to the functional replacement of the rotator cuff with an efficient work of the deltoid muscle ${ }^{1}$. The lateralizing effect of the used implant increases the action of this muscle that in some cases could be hypotrophic. Proper rehabilitation requires recovery times ranging from 3 to 6 months for patients in the Group A and B and up to 12 months for the most of cases in the Group $C^{27}$.

Indeed the final result is correlated to the rigidity of the capsular structures and muscle tendon at the time of surgery $21,14,26$.

Recent studies demonstrate that improving the function seems to be stable for at least three years, although previous studies have shown that improvements can be maintained for much longer5, 9, 24. After a reverse shoulder prosthesis is rather high the risk of implant dislocation ${ }^{10-12}$, especially linked to poor and slow healing of the repaired soft tissues; a good rehabilitation must prevent this event and respect the biological stage progressing gradually, especially in Group C patients, where deltoid muscle slowly recovers its trophy and length, after months and years of proper activity (Fig. 2).

During surgery when subscapularis tendon status allows, we repair the tendon with a tendon to tendon technique; this procedure can increase anterior stability of the implant in deltopectoral approach surgery by increasing the thickness of the soft tissue between the skin and prosthesis, according to some Authors but this issue deserve to be better studied in the future $3,4,25,27$.

Vourazeris et al. ${ }^{27}$ and Clark et al. ${ }^{3}$ did not find a significant difference in dislocations and complication rate among patients with subscapularis repair and without, on the contrary, Edwards et al. ${ }^{25}$ concluded that all their patients with an implant dislocation did not undergo to a SSC tendon repair, but all of these had a complex shoulder pathology status, like proximal humeral nonunion, fixed glenohumeral dislocation, and failed prior arthroplasty.

Nowadays, it depends largely on the implant lateralized designs that increases the lateral humeral and glenoid offsets and the deltoid provides the correct tension by increasing the compressive forces. Surely the reattachment of the subscapularis tendon delays the recovery of external rotation, so the external rotation restoration is indeed begun not before 4-6 weeks ${ }^{3,9}$. 
Reverse shoulder arthroplasty patient personalized rehabilitation protocol. Preliminary results according to prognostic groups

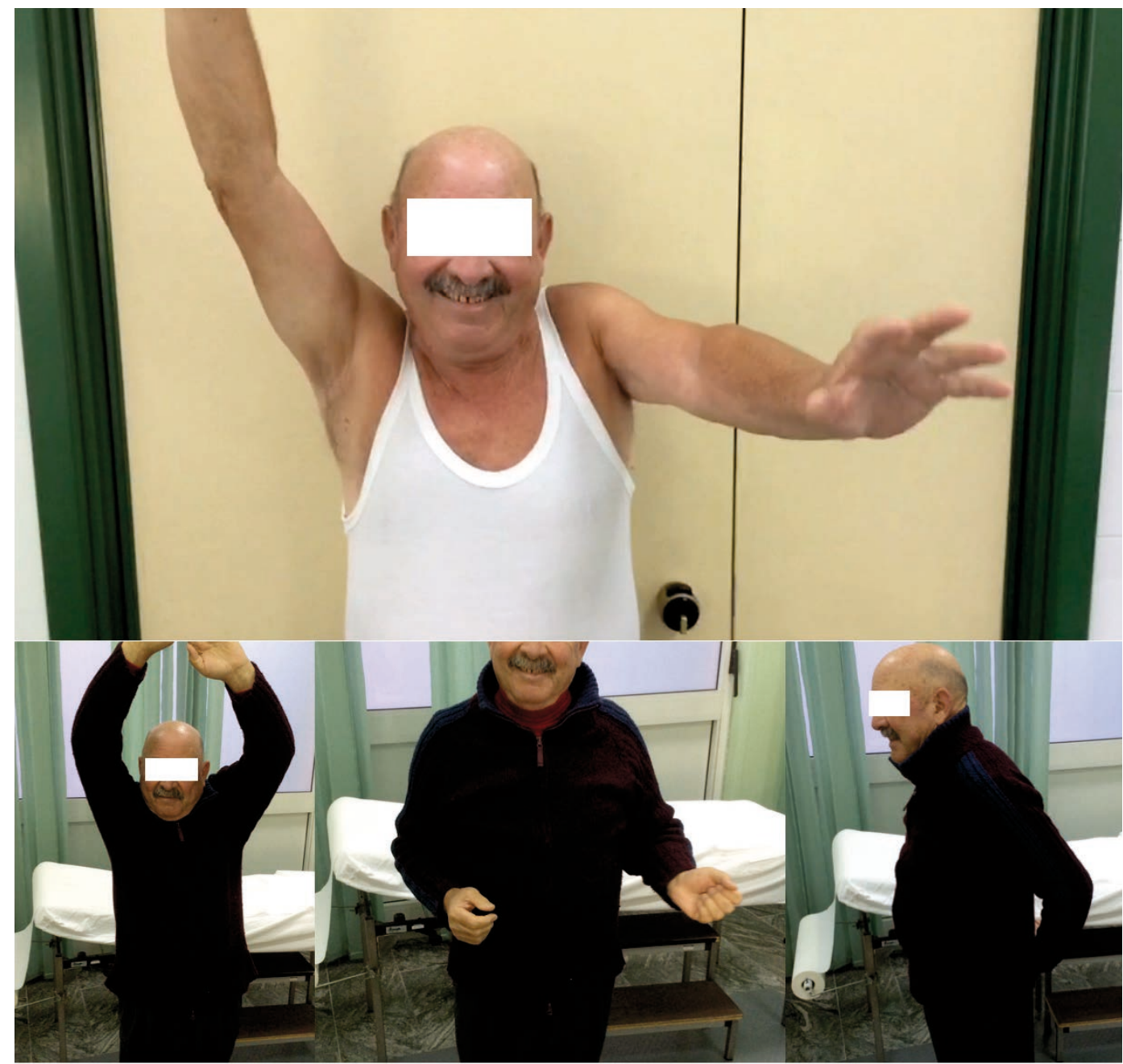

Figure 1. A high care Group C patient: preoperative (top) and postoperative ROM (below).

One of key points in the rehabilitation is the management of scapulothoracic joint 20,23 ; during that period, it is necessary for the patient to regain a correct scapulothoracic rhythm and keep it trying to avoid diskynesia. According to the surgeon indications, the physiotherapist has to prevent premature activation of the joint that would benefit not to the full recovery of the glenohumeral ROM and compromise final result. This kind of physiotherapy can also avoid possible impingement by transferring part of the humeral glenohumeral mobility to the scapulothoracic joint ${ }^{23}$.

In patients affected by hyperkyphosis, there is a reduction of the subacromial space: it can be attributed to an association of reduction of the scapular tilting with a dyskinesia of its movement. The malposition of the scapula can cause, in addition to an incorrect glenoid version and inclination intraoperative evaluation, a limitation of the recovery of ROM after RSA, caused by an abnormal orientation of the acromion at the level of scapula-acromion-clavicular chain ${ }^{20}$.

Patients with primary or cuff tear osteoarthritis or irreparable massive rotator cuff tear generally have better results (CS, ROM, satisfaction rate), compared to patients operated after fractures sequelae or revision implant; moreover, the latter have higher complication rates than patients with other etiologies ${ }^{2,} 9$.

Pazients affected by RA, Levigne stages seem to correlate with final functional outcomes; worse erosion is correlated to worse results ${ }^{19}$.

The fatty infiltration of the supraspinatus, subscapularis, infraspinatus and teres minor atrophy, the degree of preoperative head subluxation and glenoid erosion is negatively correlated with the limitation of the preoperative ROM (elevation, abduction) ${ }^{16,18}$. After implantation of the reverse prosthesis in patients with RA, they have a significant improvement in functional outcomes ${ }^{6}$.

Treatment of post-traumatic fracture sequelae of the proximal part of the humerus with malunion of the tuberosities is a challenge for any shoulder surgeon. Even when technically achieved, the published results are often unsatisfactory with anatomic replacement $^{2}, 4,28$. On the basis of the negative experience with anatomic shoulder arthroplasty, we prefer re- 


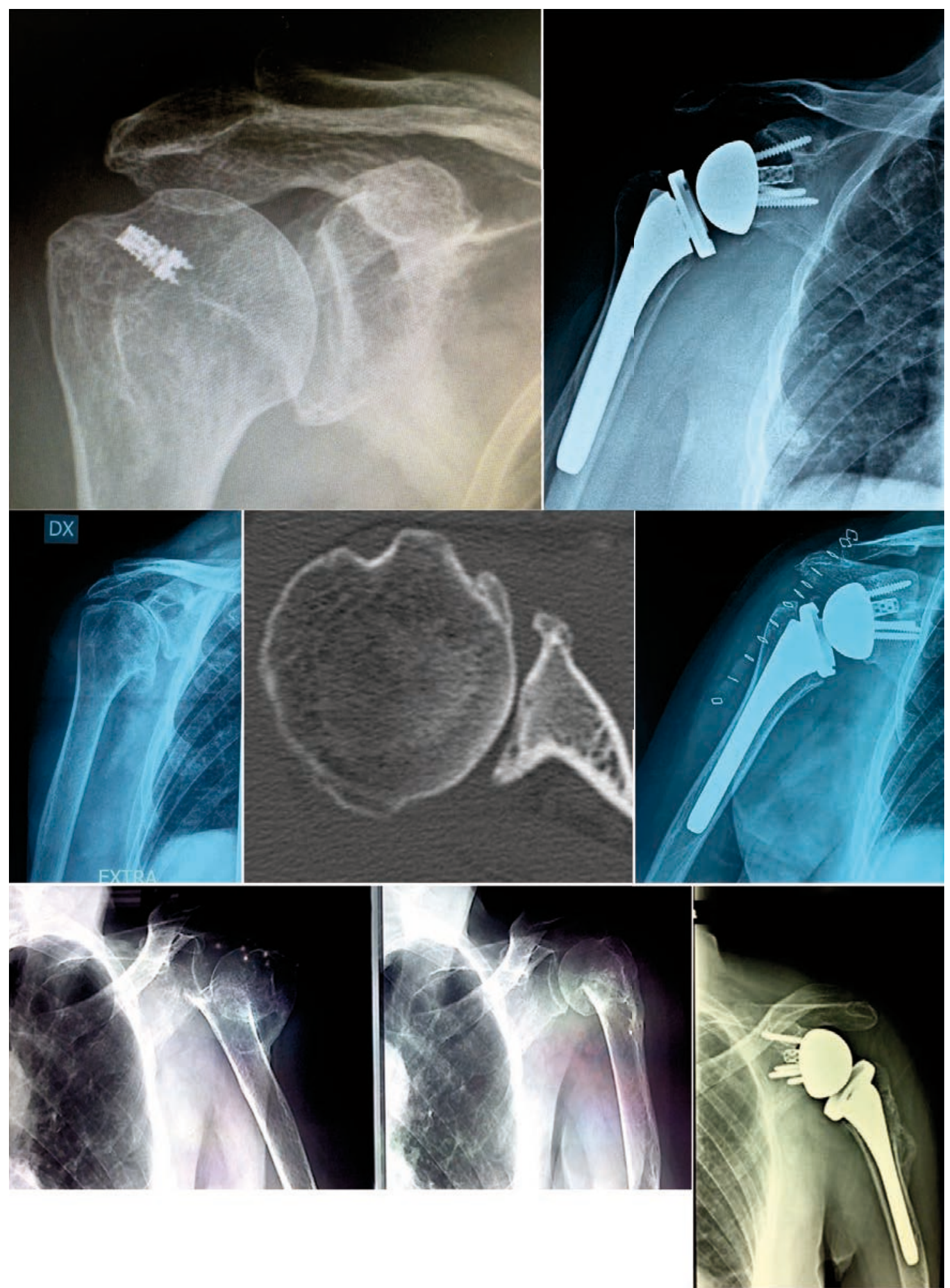

Figure 2. Preoperative and postoperative X-Rays follow-up of each group: from left to right, Group A, B, C examples, respectively.

verse shoulder arthroplasty for this condition and results can improve with a careful but intense rehabilitation, as we show in our Group C findings (Fig. 1).
Residual shoulder pain remains a less commonly observed problem following shoulder surgery. This pain is usually related to residual stiffness (in some cases 
Reverse shoulder arthroplasty patient personalized rehabilitation protocol. Preliminary results according to prognostic groups

this may be apparent by only a $10^{\circ}$ discrepancy in motion between the operated and non-operated sides), but it almost always resolves ${ }^{14}$.

\section{Study limitations}

Limitation of our study is due to its retrospective nature, short follow up duration and lacking of control goups. Although, we tried to consider three groups according to complication risk factors and establish a protocol, we do not have a real comparison among patients treated with our personalized rehabilitation protocol and a general rehabilitation program.

\section{Conclusion}

Rehabilitation after RSA is still unknown, there are only expert opinions with no evidences based proofs. It is arduous to establish universal parameters for rehabilitation protocol because we treat different patients, diagnoses, intraoperative parameters and different clinical outcomes.

Our study shows that include patients in different prognostic groups, giving them personalized protocols and early mobilization sling discharged may improve considerably clinical outcomes, as well after one year of follow up, and decrease complications rate. It demonstrated particularly worthy in difficult management patients (the high risk Group C), who dramatically improved after RSA.

\section{Conflict of interest}

Alfonso Maria Romano, Angelo Di Giunta and Massimiliano Susanna receive fees for consulting from Exactech. The other Authors declare no conflicts of interest related to the subject of this article.

This project does not infringe the Italian ethical rules and the privacy of the patients.

\section{References Manca ref. Padulo}

1. Boileau P, Watkinson DJ, Hatzidakis AM, Balg F. Grammont reverse prosthesis: design, rationale, and biomechanics. J Shoulder Elbow Surg. 2005;14(1 Suppl S):147S-161S.

2. Boileau P, Chuinard C, Le Huec JC, Walch G, Trojani C. Proximal humerus fracture sequelae: impact of a new radiographic classification on arthroplasty. Clin Orthop Relat Res. 2006; 442:121-130.

3. Clark JC, Ritchie J, Song FS, Kissenberth MJ, Tolan SJ, Hart ND, Hawkins RJ. Complication rates, dislocation, pain, and postoperative range of motion after reverse shoulder arthroplasty in patients with and without repair of the subscapularis. J Shoulder Elbow Surg. 2012;21:36-41.

4. Dines DM, Warren RF, Altchek DW, Moeckel B. Posttraumatic changes of the proximal humerus: malunion, nonunion, and osteonecrosis. Treatment with modular hemiarthroplasty or total shoulder arthroplasty. J Shoulder Elbow Surg. 1993;2 (1):11-21.

5. Guery J, Favard L, Sirveaux F, Oudet D, Mole D, Walch G. Reverse total shoulder arthroplasty. Survivorship analysis of eighty replacements followed for five to ten years. J Bone Joint
Surg Am. 2006;88:1742-1747.

6. Holcomb JO, Hebert DJ, Mighell MA, et al. Reverse shoulder arthroplasty in patients with rheumatoid arthritis. J Shoulder Elbow Surg. 2010;19:1076-1084.

7. Oliva F, Osti L, Padulo J, Maffulli N. Epidemiology of the rotator cuff tears: a new incidence related to thyroid disease. Muscles Ligaments Tendons J. 2014;4(3):309-314.

8. Sirveaux F, Favard L, Oudet D, Huquet D, Walch G, Molé D. Grammont inverted total shoulder arthroplasty in the treatment of glenohumeral osteoarthritis with massive rupture of the cuff. Results of a multicentre study of 80 shoulders. J Bone Joint Surg Br. 2004;86:388-395.

9. Wall B, Nové-Josserand L, O'Connor DP, Edwards TB, Walch G. Reverse Total Shoulder Arthroplasty: A Review of Results According to Etiology. J Bone Joint Surg Am. 2007;89:14761485.

10. Alentorn-Geli E, Samitier G, Torrens C, Wright TW. Reverse shoulder arthroplasty. Part 2: Systematic review of reoperations, revisions, problems, and complications. Int J Shoulder Surg. 2015;9:60-67.

11. Boileau P. Complications and revision of reverse total shoulder arthroplasty Orthopaedics \& Traumatology: Surgery \& Research. 2016;102(1):S33-S43.

12. Zumstein MA, Pinedo M, Old J, Boileau P. Problems, complications, reoperations, and revisions in reverse total shoulder arthroplasty: A systematic review. J Shoulder Elbow Surg. 2011;20:146-157.

13. Boudreau S, Boudreau ED, Higgins LD, Wilcox RB 3rd. Rehabilitation following reverse total shoulder arthroplasty. J Orthop Sports Phys Ther. 2007;37(12):734-743.

14. Liotard JP, Edwards TB, Padey A, Walch G, Boulahia A. Hydrotherapy Rehabilitation After Shoulder Surgery Techniques in Shoulder \& Elbow Surgery. 2003;4(2):44-49.

15. Oliva F, Piccirilli E, Bossa M, et al. I.S.Mu.L.T - Rotator Cuff Tears Guidelines. Muscles Ligaments Tendons J. 2016;5(4): 227-263.

16. Bercik MJ, Kruse K2nd, Yalizis M, Gauci MO, Chaoui J, Walch G. A modification to the Walch classification of the glenoid in primary glenohumeral osteoarthritis using three-dimensional imaging. J Shoulder Elbow Surg. 2016;25(10):1601-1606.

17. Favard L, Lautmann $S$, Clement P. Osteoarthritis with massive rotator cuff-tear: the limitation of its current definitions. In: Walch G, Boileau P, editors. Shoulder arthroplasty. Heidelberg: Springer. 1999;261-265.

18. Goutallier D, Postel JM, Bernageau J, Lavau L, Voisin MC Fatty infiltration of disrupted rotator cuff muscles. Rev Rhum Engl Ed. 1995;62(6):415-422.

19. Levigne and Franceschi Stages of Glenoid wear in RA Classification In: Shoulder Arthroplasty. Edited by Walch and Boileau. 2015;221-230.

20. Gumina S, Di Giorgio G, Postacchini F, Postacchini R. Subacromial space in adult patients with thoracic hyperkyphosis and in healthy volunteers. Chir Organi Mov. 2008;91(2):93-96.

21. Kwaees TA, Charalambous CP. Rates of surgery for frozen shoulder: an experience in England. Muscles Ligaments Tendons J. 2016;5(4):276-279.

22. Osti L, Buda M, Del Buono A. Fatty infiltration of the shoulder: diagnosis and reversibility. Muscles Ligaments Tendons $\mathrm{J}$. 2014;3(4):351-354.

23. Terrier A, Scheuber P, Pioletti PD, Farron A. Activities of daily living with reverse prostheses: importance of scapular compensation for functional mobility of the shoulder. J Shoulder Elbow Surg. 2013;22;948-953.

24. Valenti PH, Boutens D, Nérot C. In: Walch G, Boileau P, Molé D, editors. 2000 Prothèses d'épaule...recul de 2 à 10 ans. Paris: Sauramps Médical. Delta 3 reversed prosthesis for osteoarthritis with massive rotator cuff tear: long term results. $2001 ; 253-259$. 
25. Rooney SI, Loro E, Sarver JJ, et al. Exercise protocol induces muscle, tendon, and bone adaptations in the rat shoulder. Muscles Ligaments Tendons J. 2015;4(4):413-419.

26. Edwards TB, Williams MD, Labriola JE, Elkousy HA, Gartsman GM, O'Connor DP. Subscapularis insufficiency and the risk of shoulder dislocation after reverse shoulder arthroplasty. J Shoulder Elbow Surg. 2009;18(6):892-896.
27. Vourazeris JD, Wright TW, Struk AM, King JJ, Farmer KW. Primary reverse total shoulder arthroplasty outcomes in patients with subscapularis repair versus tenotomy. J Shoulder Elbow Surg. 2016;S1058-2746(16)30438-4.

28. Mansat $P$, Guity MR, Bellumore $Y$, Mansat M. Shoulder arthroplasty for late sequelae of proximal humeral fractures. J Shoulder Elbow Surg. 2004;13(3):305-312. 